\title{
Hot Cracking Mechanisms in Welding Metallurgy: A Review of Theoretical Approaches
}

\author{
Dimosthenis Manitsas, Joel Andersson ${ }^{1}$ \\ ${ }^{1}$ University West, Department of Engineering Science, Trollhättan, Sweden
}

\begin{abstract}
Hot cracking often refers to the appearance of liquid films along grain boundaries or to another place in the weld metal structure. Despite hot cracking importance in alloy weldability, there is limited understanding of the influencing mechanisms. Theories and criteria worked out over the years to assess alloy weldability will be presented. The review focuses on: 1) Theories of hot cracking, 2) Hot cracking criteria, and 3) A criticism of hot cracking theories and criteria.
\end{abstract}

\section{Introduction}

It is known that some weld metal hot cracking theories have its origins in castings. However, it is critical to emphasize that casting processes have important differences as compared to weld processes, like time and length ratios. The cracking during welding can be attributed to the effect of tensile stresses on a not well developed dendritic network in the weld metal and/or heat affected zone (HAZ). Previously published works documented the different types of cracking by the temperature variation within they occur [1]. "Hot cracking" is related to the appearance of liquid films along grain boundaries or to another location in the structure and can be divided into 1) solidification cracking in the weld metal, 2) liquation cracking in the HAZ, and 3) weld metal liquation cracking [1]. This is then followed by "warm cracking". Warm cracking includes 1) ductility-dip cracking, 2) reheat cracking, 3) strain age cracking, and 4) lamellar cracking [1]. Warm cracking can appear either during the welding process or after post weld heat treatment. Last, but not least, "cold cracking" develops at room temperature because of the presence of hydrogen and hydrogen-assisted cracking mechanisms [1]. This review presents the five theories, which are being used to study solidification cracking in welding metallurgy. The five theories of hot cracking formation include 1) shrinkage-brittleness theory, 2) strain theory, 3) generalized theory, 4) modified generalized theory, and 5) technological strength theory. Based on these five main hot cracking theories, criteria for cracking during solidification in welding have been developed over the past years. Additionally, factors governing the solidification cracking in the weld metal and the critera for cracking susceptibility are also summarized.

\section{Background}

\subsection{Hot cracking theories}

\subsubsection{Shrinkage-brittleness theory}

In 1948 Pumphrey and Jennings [2] stated the "shrinkage-brittleness theory". The shrinkage-brittleness theory address the importance of an "effective interval" of solidification, which exists at a lower temperature than "coherency" temperature, as illustrated in Figure 1.

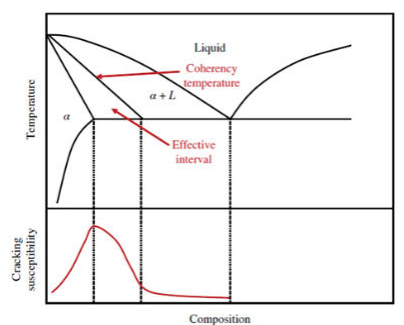

Fig. 1. Schematic illustration of the "shrinkage-brittleness theory" [3]

Also, shrinkage-brittleness theory introduces the "healing effects" of the residual liquid. The theory considers that the primary dendrites come into contact for first time at the coherency temperature. The theory hypothesizes that the brittle temperature range (BTR) is between the solidus and coherency temperature where the weld metal 
has low ductility. Solidification cracking will occur in the BTR, when shrinkage strains exceed a critical level. Cracking will appear after solid-solid bridging, which allows strain to build in the structure. At a temperature, range above the effective interval the remaining liquid is adequate to heal cracks that may occur. Moreover, micro-segregation is important, since the diffusion of solute into the solid phase during welding is small. Microsegregation is increased and in accordance with the shrinkage-brittleness theory, low melting temperature point elements increase the hot cracking susceptibility. Cracking susceptibility will be highest at the composition of the alloy, where the BTR is the widest.

\subsubsection{Strain theory}

In 1954 Apblet and Pellini [4] stated the "strain theory". Strain theory of hot cracking introduces the concept of strain accumulation and continuous liquid film, which is present at the solidification stages. The strain theory claims that solidification consists of two stages the 1) mushy zone, and 2) liquid film stage. Within this framework, the temperature region, where the mushy zone occurs is higher than the liquid film stage. The mushy zone of the alloy is a two-phase mixture, namely the mushy zone consisting of a liquid and solid phase, which means that the alloy has a large liquid fraction. The liquid film is thick; hence, solidification cracks do not appear in the mushy zone owing to the uniform strain distribution. Strain starts to build up during the film stage of solidification. The liquid film stage has the early and late period. Before complete solidification, or at the late film stage, a thin liquid film is present, which separates the solid grains. Figure 2 shows the temperature, the film stage time and the strain rate in accordance with the strain theory. It is visible that the life of the normal film stage is smaller than that of segregate film stage. Apblet and Pellini [4] suggested that the equilibrium solidification is described by the normal film stage. On the other hand, segregation broaden the time of the liquid film stage delaying the solidification process. The liquid film become thinner and thinner under nonequilibrium solidification conditions. Consequently, the thin liquid film cannot accomplish the developed tensile strain at the grain boundary, resulting from shrinkage and thermal contraction. When the strain exceeds a critical value, cracks will appear.

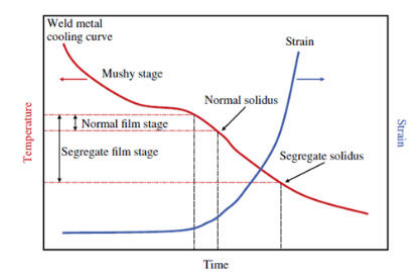

Fig. 2. Schematic illustration of the "strain theory" [3]

A shrinkage defect appears in a hot spot in welding, where the thermal gradient is smaller than the critical value, or when a critical solid fraction is reached. This infers the finding that cracking susceptibility is affected by the time, temperature and the amount of the interdendritic liquid.

\subsubsection{Generalized theory}

In 1960 Borland [5] postulated the "generalized theory". On one hand, generalized theory is an alteration of the shrinkage-brittleness theory, and on the other contained some strain theory features. Borland has suggested four solidification steps, namely, 1) the primary dendrite formation, 2) the dendrite interlocking, 3) the grain boundary development, and 4) the complete solidification. Within this framework, (1) the primary dendrite development (mushy stage), the liquid is continuous and the solid phase is scattered in it. Both of the phases are able to move, so cracking do not occur. (2) At the dendrite interlocking stage (coherency temperature, coherent temperature range), both the solid and liquid phase is continuous, however, only the liquid phase is able to move and to flow between the dendrites. At the dendrite interlocking stage, if the grains separates because of the strain, the interdendritic liquid will heal any cracks. (3) At the grain boundary stage, the solid network is well developed and coalesced, forming solid-solid bridges, which do not allow the liquid phase to flow between them. Borland defined it as the "critical solidification range". At the "critical" range, the cracks cannot "recover" because of the reduced amount of the liquid, which means that the liquid volume cannot be interconnected. (4) Finally, the solidification is completed, and no solidification cracking occurs, owing to the lack of liquid. Furthermore, Borland studied how the hot cracking susceptibility is influenced not only by the quantity of the liquid phase, but also by the liquid distribution at grain corners, edges and faces. The distribution of the liquid phase is estimated by the interfacial energy ratio or liquid wettability $\tau$ on grains in accordance with the following equation: 


$$
\tau=\frac{\gamma_{S L}}{\gamma_{S S}}=\frac{1}{2 \cos (\varphi / 2)}(1)
$$

Where $\gamma_{S L}$ is solid-liquid interface energy, $\gamma_{S S}$ is energy of solid-solid interface, and $\varphi$ is the dihedral wetting angle, which is between $0^{\circ}$ and $180^{\circ}$. When the ratio of the interphase boundary tension and the grain boundary tension $\left(\gamma_{\mathrm{SL}} / \gamma_{\mathrm{SS}}\right)$ is equal to 0.5 or below $\left(\varphi<60^{\circ}\right)$, there is a complete de-cohesion between the solid grains, since a continuous liquid channel wet the grain boundaries. This explains why the cracking susceptibility is low at the mushy stage. As the ratio increases, the dihedral wetting angle increases, also the liquid phase degree by degree will cover less grain faces. At an angle of $60^{\circ}$ the liquid-solid interface is decreased, which means that only a continuous liquid film remains along grain edges. At values of dihedral wetting angles higher than $60^{\circ}$, but less than $90^{\circ}$, liquid phase will remain as isolated droplets at grain corners. When solid-solid bridges formed, slightly above $90^{\circ}$, the cracking susceptibility is high owning to high stresses. At even higher dihedral angles more dendrites bridges exist, and hence the susceptibility to cracking is reduced. From the foregoing, it is easy to see that the distribution of the liquid phase along grain boundaries influences the strength to fracture.

\subsubsection{Modified generalized theory}

In 1982 Matsuda et al [6] employed the shrinkage-brittleness and generalized theories on in-situ observation of the solidification front and solidification cracking in the weld metal. They reported the "modified generalized theory". As illustrated at Figure 3 they suggested three different stages of cracking susceptibility: 1) The liquid mass stage, where the solid phase is totally wrapped by thick liquid film. The coherency temperature appears at the final part in the liquid mass stage. 2) The liquid film stage, the secondary growth of dendrites is shown in an early stage of solidification, below the liquidus temperature. Which means that the second stage occurs in a more limited temperature range than originally mentioned. At the liquid film stage, the residual liquid films have low dihedral angles on grain boundaries, which are formed by the dendritic network. The critical solidification stage appears at the highest temperature limit of the liquid mass stage. In other words, the critical temperature is at the border between the liquid mass and the liquid film stage. 3) The liquid droplet stage is longer than the first two stages, as illustrated in Figure 3. It is named as the liquid droplet stage owing to the scattered residual liquids. The third period is divided into two stages, namely the $3 \mathrm{H}$ and $3 \mathrm{~L}$. According to modified generalized theory the stage $3 \mathrm{H}$ (thin continuous liquid film) is susceptible to crack initiation, and the stage 3L (isolated liquid droplets) is not susceptible to crack initiation, but to crack propagation. At the droplet stage, the crack initiation is impossible due to the formation of coalesced solid-solid bridges.

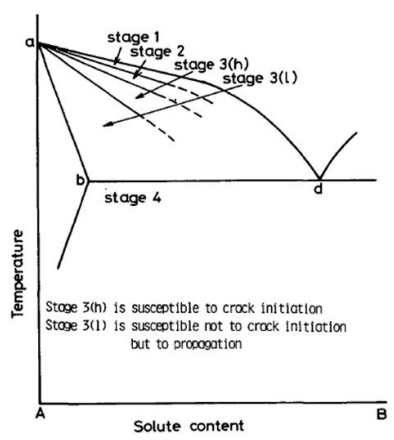

Fig. 3. Relationship between solidification path and hot cracking according to the "modified generalized theory"

Matsuda and his team [7] reported a metallographic and fractographic analysis of solidification crack in weld metal using SEM. They mentioned four different failure types in accordance with crack surface observations. Figure 4 illustrates the initiation of the crack temperature, where the temperature is lowered from the left to the right side: (1) Type D: The crack surface at the highest temperature region, close to liquidus temperature, has a dendrite morphology. It is easily understood that the initial letter of "dendrite surface" have named the Type D region. (2) Type F: The crack surface at the lowest temperature region has a flat characteristic appearance, thus is named Type F. In Type F region, the solid network is in progress, as the temperature falls and the residual liquid droplets are reduced. Also, in Type F region, the grain boundary starts to move at solid network parts. Cracking propagation occurs at the migrating grain boundaries connecting the scattered liquid droplets. The ductility increases steadily in Type F due to the temperature fall and the coalesce of the solid-solid bridges. (3) Type F+D: The crack surface at the medium temperature region has a feature of coarse dendrites, as the 
secondary dendrites come close to each other, since the interlocking process begins. In other words, it illustrates the alteration from dendritic to flat fracture. This region is named Type $\mathrm{F}+\mathrm{D}$ region, since it shows the passing from Type D to Type F. Another point worth mentioning is that the ductility is low, as the liquid films are still placed around the grain boundaries. (4) Type R: A rough crack surface named this type of failure, which is an intergranular, solid-state fracture. Ductility-dip cracking is pictured by the Type R failure.

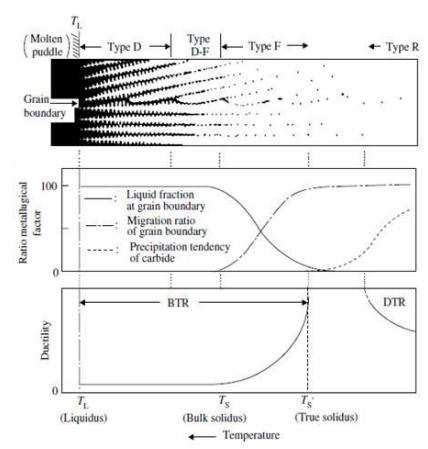

Fig. 4. Relationship between solidification process, liquation fraction at grain boundary, migration ratio of grain boundary and ductility of weld metal [3]

\subsubsection{Technological strength theory}

At the latter stages of welding solidification, cracks appear because of the increase in tensile shrinkage stress and the liquid films existence along the solidifying grain boundaries in the metal structure. As a result, cracking will occur when the enforced shrinkage strain is greater than the inherent ductility of the solidifying weld metal. According to Prokhorov [8] and Senda et al [9] the temperature limits within which solidification cracking takes place described as BTR or as Critical Strain rate for Temperature drop (CST). There is an evident analogy between the two theoretical concepts in which strain rate is a noteworthy characteristic as describes the ability of the metal to gather deformations without tearing during solidification. The BTR is symbolized by a fall in ductility (depth) between the liquidus and solidus temperature (width). There are three aspects regarding the requirements for the solidification cracking in the weld metal according to Prokhorov's model (1) The brittle temperature range of the weld metal during solidification, (2) The minimum ductility of the metal in the brittle temperature range and (3) the rate of deformation of the solidifying metal in the brittle temperature range. Taking these considerations into account, it is understandable that the critical rate of stain accumulation is the limit between solidification crack and no crack qualifications. Solidification cracking will appear in the weld metal if the rate of deformation surpasses the ductility curve in the BTR. The less deep and wide the BTR, the more resistant the weld metal is to cracking. The rate of strain accumulation with temperature fall $(\mathrm{d} \varepsilon / \mathrm{dT})$ is connected with the cooling rate $(\mathrm{dT} / \mathrm{dt})$ and the strain rate $(\mathrm{d} \varepsilon / \mathrm{dt})$, as follows:

$$
\mathrm{d} \varepsilon / \mathrm{dT}=(\mathrm{d} \varepsilon / \mathrm{dt}) /(\mathrm{dT} / \mathrm{dt})(2)
$$

The physical clarification of this ductility based model is that the semi-solid weld metal at the latter stage of solidification can be fractured because of the plastic deformation, which is caused by diffusion creep, imposed thermal strains, and/or microstructural rearrangement. The outgrowth of the technological strength theory is that there are a thermal and mechanical characteristic, namely the weld metal cooling rate and the ductility of the mushy zone, which affect the minimum strain rate for solidification cracking.

\subsection{Hot cracking criteria}

\subsubsection{Reserve of plasticity criterion}

Novikov et al [10] mentioned another approach of the hot cracking susceptibility, as they introduced the variable of "reserve of plasticity" $p_{r}$ in the solidification range. Novikov et al [10] pointed out that the smaller the ductile in the brittleness temperature range and the larger the linear shrinkage in the effective interval, the greater is the solidification cracking susceptibility in the weld metal. There have been two parameters that have been assisted in understanding the role of reserve of plasticity in the solidification range. (1) The plastic deformation mechanism is based on the grain boundary movement along the liquid films, which are around the grains in the pre-eutectic condition. The thicker the liquid films are the greater the elongation to failure is. (2) Decreased grain size assists the progress of grain boundary movement, therefore the elongation to failure in the 
solidification range becomes easier. Elongation to failure in the solidification range is proportionate to the linear shrinkage in the crystallization range according to reserve of plasticity model. The effective interval of crystallisation can be estimated by the following equation:

$$
\varepsilon_{c r}=\alpha_{\mathrm{a}} \times \Delta T_{e f} \times 100
$$

Where $\alpha_{\mathrm{a}}$ is the average thermal coefficient of linear shrinkage and $\Delta \mathrm{T}_{\text {ef }}$ is the effective interval of crystallisation.

The reserve of plasticity $\mathrm{p}_{\mathrm{r}}$ is estimated by the following equaton:

$$
p_{r}=\frac{1}{\Delta T_{b r}} \int_{T_{c o h}}^{T_{s o l}}\left(\varepsilon_{p}-\varepsilon_{s h}\right) d T=\frac{S}{\Delta T_{b r}}(4)
$$

Where $S$ is the area between the ductility curve $\varepsilon_{\mathrm{p}}$ from the solidification shrinkage curve $\varepsilon_{\mathrm{sh}}$ in the brittle temperature range $\Delta \mathrm{T}_{\mathrm{br}}$ hence between coherency $\mathrm{T}_{\text {coh }}$ and solidus $\mathrm{T}_{\text {sol }}$ temperatures.

\subsubsection{Solidification shrinkage $\&$ interdendritic melt flow criteria}

In 1977 Feurer [11] focused on entirely metallurgical, non-mechanical justification of solidification cracking in the mushy zone. During plane front or cellular solidification, shrinkage stresses appear due to the remaining liquid, which is in front of the solid-liquid interface. The mass feeding is not easy during dendritic solidification. At the latter stage of the dendritic solidification the dendrites come into contact with one another, and a fixed solid network occurs. The passage from one state to another is connected with a shear strength expansion and with a further decrease in feeding. At this time, phenomena like macro-segregation, macro- and micro-shrinkage start to form, while only interdendritic feeding is possible. If the rate of feeding (ROF) is greater or equal to the rate of shrinkage (ROS) cracks will not occur (Figure 5). The rate of the liquid feeding in the mushy region cannot counterbalance the effects of shrinkage and thermal stresses. In other words, solidification cracking in the weld metal takes place when the rate of shrinkage (ROS) is greater than the rate of feeding (ROF).

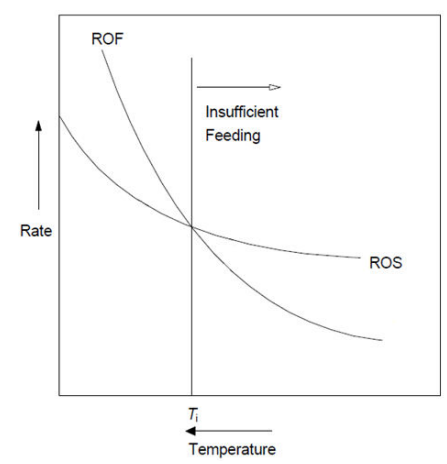

Fig. 5. Schematic illustration of rate of feeding and rate of shrinkage according to Feurer's model [11]

The rate of the liquid feeding can be calculated according to Darcy law, which explains the fluid flow through a porous material. Darcy law describes the mean interdendritic velocity to the pressure gradient, with a measurable factor, which is proportional to the viscosity. On the other hand, the rate of shrinkage is controlled by the coalescence of the dendritic solid network with temperature during solidification, which is affected by the segregation attitude of the alloy. It could be claimed that Feurer's criterion is influenced by cooling rate and chemical composition of the alloy in a consistent way.

$$
R O F=\left(\frac{\partial \ln V}{\partial t}\right)_{\text {feeding }}=\frac{f_{l}^{2} \lambda_{2}^{2} P_{S}}{24 \pi c^{3} \mu L^{2}}(5)
$$

Where

$$
P_{S}=P_{O}+P_{M}-P_{C}(6)
$$

Where $\mathrm{P}_{\mathrm{s}}$ is effective feeding pressure, $\mathrm{P}_{\mathrm{o}}, \mathrm{P}_{\mathrm{M}}$, and $\mathrm{P}_{\mathrm{C}}$ are atmospheric, metallostatic and capillary pressure respectively, $f_{1}$ is volume of liquid in the dendrite network, $c$ is tortuosity constant of the dendritic network, $L$ is 
the size of the mushy zone, $\mu$ is viscosity of the liquid phase, $\lambda_{2}$ is secondary dendrite arm spacing, $\mathrm{V}$ is volume, and $t$ is time.

Where

$$
R O S=\left(\frac{\partial \ln V}{\partial t}\right)_{\text {shrinkage }}=-\frac{1}{\bar{\rho}} \frac{\partial \bar{\rho}}{\partial t}=\frac{\left(\rho_{O}-\rho_{S}+\alpha k C_{L}\right) \dot{T} f_{L}^{2-k}}{\rho(1-k) m_{L} C_{O}}
$$

$$
\begin{gathered}
P_{M}=\bar{\rho} g h \\
\bar{\rho}=\rho_{L} f_{L}+\rho_{S} f_{S}=\rho_{L} f_{L}+\rho_{S}\left(1-f_{L}\right) \\
P_{C}=\frac{4 \gamma_{S L}}{\lambda_{2}}
\end{gathered}
$$

Where $\gamma_{\mathrm{SL}}$ is solid liquid interfacial energy, $\mathrm{g}$ is gravity constant, $\mathrm{h}$ is distance to the melt surface, $\bar{\rho}$ is average density, $\rho_{\mathrm{L}}$ is the density of the liquid phase, $\rho_{0}$ is the density of liquid at the melting point, $\rho_{\mathrm{S}}$ is the density of the solid phase, $\alpha$ is the composition coefficient of liquid density, $\mathrm{C}_{\mathrm{L}}$ is the composition of the liquid at the solid-liquid interface, $\mathrm{C}_{0}$ is the alloy composition, $\varepsilon$ is the average cooling rate during solidification, $\mathrm{k}$ is the equilibrium partitioning coefficient, $f_{1}$ and $f_{s}$ are the volume fractions of liquid and solid in the dendritic network, and $\mathrm{m}_{\mathrm{L}}$ is the slope of the liquidus line. In 1981 Clyne and Davies [12] postulated a hot cracking criterion based on the generalised theory (grain boundary development step) and Feurer's model at the latter step of solidification. Clyne and Davies related the solidification cracking susceptibility and the chemical composition of binary alloys with the existence of a critical time period, when the interdendritic liquid cannot flow freely and the produced strains cannot be recovered by the rate of feeding. Thus, at that period the solidsolid network is easily acceptable on cracking. Clyne and Davies model is based on a cracking susceptibility coefficient (CSC), namely is a ratio of the vulnerable time period, where the dendritic arms can easily be separated $t_{v}$, and the time available for stress heal, $t_{r}$. The ratio $t_{v} / t_{r}$ is the cracking susceptibility coefficient, where (1) $t_{v}$ is the vulnerable time period in $0.90<f_{s}<0.99$ and (2) $t_{r}$ is the time available for stress heal in 0.40 $<\mathrm{f}_{\mathrm{s}}<0.90$. Another interesting aspect of the Clyne and Davies's criterion is that the times were calculated at various cooling rates in casting.

\subsubsection{Interdendritic pressure drop, critical rate of perpendicular thermal strain \& solidification shrinkage criterion}

The RDG hot cracking criterion of Rapaz, Drezet, and Gremaud [13] suggested in 1999. RDG criterion adds together the grain boundary development, rate of liquid feeding in the mushy zone, and a model at which the beginning of a crack in the interdendritic liquid exists. RDG model can be explained by the highest vallue of a strain rate $(\dot{\varepsilon} \max )$ that the mushy zone can undergo before cracking occurance. The inverse value of the maximum deformation rate $\left(\dot{\varepsilon}^{-1}{ }_{\max }\right)$ is the meaning of a hot cracking susceptibility (HCS) at the dendrite root, according to RDG criterion. RDG model indicate that a solidification cracking in the weld metal is created when the interdendritic liquid (metallostatic) pressure $\left(\mathrm{p}_{\mathrm{m}}\right)$ becomes lower than cavitation pressure $\left(\mathrm{p}_{\mathrm{c}}\right)$, a void or a gas pore will form, acting as crack nuclei. From the RDG model, it can be considered that the presssure falls because the solid bridge bonding is deficient in liquid feeding to accommodate tensile deformation perpendicular to the direction growth and solidification shrinkage. Thus, it can be reasonably deducted that a hot crack will be produced at the critical pressure $\left(\mathrm{p}_{\min }\right)$ or the maximum pressure drop $\left(\Delta \mathrm{p}_{\max }\right)$. The maximum pressure drop can be estimated by the following equation:

$$
\begin{gathered}
\Delta p_{\max }=\Delta p_{\varepsilon}+\Delta p_{s h}=p_{m}-p_{c}= \\
\frac{180}{\lambda^{2}} \frac{(1+\beta) \mu}{G} \int_{T_{S}}^{T_{L}} \frac{E(T) f_{S}(T)^{2}}{\left(1-f_{S}(T)\right)^{3}} d T+\frac{180}{\lambda^{2}} \frac{v_{T} \beta \mu}{G} \int_{T_{S}}^{T_{L}} \frac{f_{S}(T)^{2}}{\left(1-f_{S}(T)\right)^{2}} d T(11)
\end{gathered}
$$

Where $E(T)=\frac{1}{G} \int f_{S}(T) \dot{\varepsilon}_{P}(T) d T, \beta$ is shrinkage factor, $\mu$ is liquid viscosity, $\lambda$ is secondly dendrite arm spacing, $G$ is thermal gradient, $v_{T}$ is isotherms velocity, $f_{s}(T)$ is solid fraction at any temperature $T, T_{L}$ is liquidus temperature and $T_{s}$ is coherency temperature. The cavitation pressure is set as $2 \mathrm{kPa}$ for Al-Cu alloys and almost $90 \mathrm{kPa}$ for stainless steel. However, it is critical to emphasize that the cavitation pressure is an unidentified value of RDG model. 


\subsubsection{Grain boundary, intergranular liquid flow, \& lateral tensile deformation rate criterion}

In 2015 Kou [14] employed the existent solidification theory and transport phenomena on a solidification cracking criterion. The scope of Kou's study was to report an index for cracking susceptibility of an alloy including 1) columnar dendritic grains growth in one direction, 2) tensile deformation at the lateral side of the grains growth and 3) feeding of liquid at contrary direction of growth rate. The condition of solidification cracking according to Kou's study is written as:

$$
\left\{\frac{d \varepsilon_{\text {local }}}{d T}>\sqrt{1-\beta} \frac{d \sqrt{f_{s}}}{d T}+\frac{1}{(d T / d t)} \frac{d}{d z}\left[\left(1-\sqrt{1-\beta} \sqrt{f_{s}}\right) v_{z}\right]\right\}_{\sqrt{f_{s}} \rightarrow 1}
$$

Where $\varepsilon_{\text {local }}$ is local tensile strain in the mushy zone, $t$ is time, $\beta$ is solidification shrinkage, $\mathrm{f}_{\mathrm{s}}$ is solid fraction during solidification, $\mathrm{T}$ is temperature, $\mathrm{z}$ is axial direction of columnar dendritic grains, $v_{\mathrm{z}}$ is velocity of liquid feeding along the grain boundary. Also, the three terms symbolize the strain rate, growth rate and feeding rate, all of them are positive. Kou's model presents that solidification cracking in the weld metal occur under three requirements: (1) Rapid stain rate $\mathrm{d} \varepsilon / \mathrm{dt}$, (2) slower cooling rate $\mathrm{dT} / \mathrm{dt}$ and (3) rapid strain accumulation rate with temperature drop $\mathrm{d} \varepsilon_{\text {local }} / \mathrm{dT}$. It should be mentioned here that the criterion shows the necessary condition for solidification cracking to happen. Kou's criterion doesn't show the sufficient condition for solidification cracking in the weld metal. A condition for solidification cracking is sufficient, when nucleation sites pre-exist, like entrapped bifilms and micro gas porosity. The index of solidification cracking is reported as $\left|d T / d\left(f_{s}\right)^{1 / 2}\right|$ near $\left(f_{s}\right)^{1 / 2}=1$. It could be claimed that a high absolute value $\left|d T / d\left(f_{s}^{1 / 2}\right)\right|$ near $\left(f_{s}\right)^{1 / 2}=1$, means that the growth term becomes smaller and hence the slower the growth rate is, the easier to occur cracking. In other words, the higher the absolute value $\left|d T / d\left(f_{s}^{1 / 2}\right)\right|$ near $\left(f_{s}\right)^{1 / 2}=1$, the smaller the liquid feeding rate along the grain boundary, that is more difficult to resist cracking. More specifically, a high $\left|d T / d\left(f_{s}\right)^{1 / 2}\right|$ near $\left(f_{s}\right)^{1 / 2}=1$ claims that $\left(d\left(f_{s}\right)^{1 / 2} / d T\right)(d T / d t)$ is low. Therefore, the growth rate of the grain bridge bonding is slow and the cracking resistance becomes more difficult. Because the grains grow and coalesce in a slow manner, the liquid chanel along the grain boundary near $\left(f_{s}\right)^{1 / 2}=1$ is lengthened. According to Poiseuille law, the rate of the volumetric flow of a liquid through a channel diminishes accompanying the increase of channel length because of the resistance to flow, which is caused by the viscosity of the liquid. In Kou's study the differential control volume does not move and is compromised by dendrite arms and the interdendritic liquid, however is stationary and it is compromised by the space at the grain boundary and the intergranular liquid. Apart from this, the differential equation of the criterion is in the form of unsteady-state and single phase. Furthermore, cracking occurs near the end of solidification $\left(f_{s}\right)^{1 / 2} \rightarrow 1$, when the rate of net space exceeds the rate of liquid feeding.

\subsubsection{Grain boundary, intergranular liquid flow, \& lateral tensile deformation rate criterion}

In 2005 Ploshikhin and his co-workers [15] studied the impact of the accumulated tensile strains in the interdendritic liquid phase of segregates at the latter stage of solidified weld metal. It has been demonstrated a thermo-mechanical and metallurgical model to estimate a solidification cracking index, which depends on nature of design (DS), welding conditions (WC), properties of the base metal (PBM), properties of the filler material (PFM), and parameters of the microstructure (PMS). The solidification cracking index can be written as:

$$
\delta_{a c c}^{\max }(D S, W C, P B M, P F M) \geq \delta_{a c c}^{c r}(P M S)
$$

Where $\delta_{a c c}^{\max }$ is maximum displacement accumulated in the interdendritic liquid plase, and $\delta_{a c c}^{c r}$ is critical displacemeent. The separation term of the left hand side of the solidification cracking criterion is associated with the thermo-mechanical aspect of the model. It should also be noted that the left hand side of the criterion presents the mechanical rate of the interdendritic liquid film in the mushy zone between liquidus and solidus temperatures. The right side of this criterion contains the metallurgical parameters. The right separation term shows the cracking susceptability of the interdendritic liquid phase of segregates, which depends on the thickness (fraction of the residual liquid) and the morphology (primary and secondary arm spacing) of the liquid phase. 


\section{Discussion}

\subsection{Limitations of hot cracking theories}

There are several limitations regarding the developed hot cracking models that were presented in this work. The main criticism belong tot he fact that it is evident that cracking susceptability of the shrinkage-brittleness theory is dependent on the alloy composition. In addition to this, it is apparent that according to the strain theory the mechanism of crack initiation is explained by a physically acceptable mechanical-metallurgical approach. Also, it is easily understood that strain theory does not point out any quantitative index for cracking susceptibility, as it was a qualitative explanation of the strain partitioning within the mushy zone. The generalized theory does not take account to the non-equilibrium segregation would increase the cooling rate for even very dilute alloys. Also, it should be pointed out that the dihedral wetting angle on cracking is not easy to determine. The technological strength theory is a mechanical dependent approach, which cannot be physically connected with any liquid rupture mechanism about solidification cracking. Also, it is approved that is not easy in practice to determine the BTR.

A negative aspect of Feurer's model is that the exact quantities for calculating the solidification cracking criterion in the mushy zone are difficult to be estimated. What is more, a common criticism of Clyne and Davies's criterion set ad hoc critical volume fractions of interdendritic liquid and solidifcation times. Consequently, it is suggested a somewhat arbitrary hot cracking criterion. Additionally, the effects of alloy microstructure and mechanical properties are not considered. However, microstructure and mechanical properties in the semi-solid state has a significant role on hot cracking susceptability. Meaning that Clyne and Davies's criterion is limited applicable on welding technology. The Rappaz-Drezet-Gremaud theory shows some limitations. In more rigorous consideration, one would say that solidification cracking propagation in the weld metal is not included in the RDG criterion. According to RDG approach the deformation strains are applied perpendicularly to the growth direction, which is not completely acceptable. It is important to note that there is either a suction or expulsion of the liquid on the longitudinal section. RDG criterion is developed upon columnar dendritic grain structure. Concerning grain boundaries RDG model does not take into account any localization of the strains. The accuracy of Kou's model depends on the choice of the solid fraction range. It is of vital importance to note that there is no theoretical basis on solid fraction choice of each alloy. The empirical selected range of the solid fraction set limitations in the use of this model.

\section{Conclusion}

A review of theoretical approaches of hot cracking mechanisms in welding metallurgy have been made in this paper. It can be noticed from the above discussion that a trustworthy hot cracking criterion able to accurately estimate the solidification cracking behavior in the weld metal under different conditions is still needed. It is inferred from the above that hot cracking can be classified into metallurgical (BTR, primary grain boundaries, porosity, liquid feeding, dendrite coherency and Interfacial tension) or mechanical factors (strain, strain rate and stress).

\section{References}

1. J. C. Lippold, Hot cracking phenomena in welds, 271-290 (2005)

2. W. I. Pumphrey, P. H. Jennings, J. Inst. Metal., 75, 235-256 (1948)

3. J. C. Lippold, Hot cracking, in Welding metallurgy and weldability, 84-129 (2015)

4. W. R. Apblett, W. S. Pellini, Weld. J., 33(2), 83s-90s (1954)

5. J. C. Borland, Br. Weld. J., 7(8), 508-512, (1960)

6. F. Matsuda, H. Nakagawa, K. Sorada, Trans. JWRI, 11(2), 67-77 (1982)

7. F. Matsuda, H. Nakagawa, S. Ogata, S. Katayama, Trans. JWRI, 7(1), 59-70 (1978)

8. N. N. Prokhorov, Weld. Product., 9(4), 1-8 (1962)

9. T. Senda, F.Matsuda, G. Takano, K. Watanabe, T. Kobayashi, T. Matsuzaka, Trans. JWS, 2(2), 141-162 (1971)

10. I. I. Novikov, O. E. Grushko, Mater. Sci. Technol. 11(9), 926-932 (1995)

11. U. Feurer, The symposium on quality control of engineering Alloys and the role of metal science (1977)

12. T. W. Clyne, G. J. Davies, Br. Foundrym., 71(4), 65-73 (1981)

13. M. Rappaz, J. -M. Drezet, M. Gremaud, Metall. Mater. Trans. A, 30A(2), 449-455 (1999)

14. S. Kou, Acta Mater., 88, 366-374 (2015)

15. V. Ploshikhin, A. Prikhodovsky, M. Makhutin, A. Ilin, H. -W. Zoch, Hot cracking phenomena in welds, 223-244 (2005) 